\title{
JUURNAL.RU
}

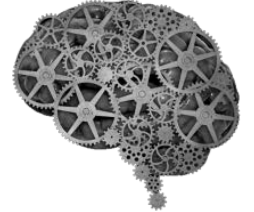

COMPANY GROUP "INTELLEKT"

\author{
Федотов А.И. ${ }^{1}$, Лисин С.К. ${ }^{2}$ \\ ${ }^{1}$ СПб политехнический университет Петра Великого \\ ${ }^{2}$ СПб наииональный минерально-сырьевой университет «Горный» \\ Санкт-Петербург, Россия
}

doi: 10.18411/lj2016-7-2-14

idsp 000001: lj2016-16-2-14

\section{Анализ аспектов моделирования и применения вибрационного контроля физико-механических свойств}

\section{Lisin Sergei}

\section{Analysis of the aspects of modeling and application} for vibration testing of physical and mechanical properties

\section{Article summary}

The article discusses aspects of modelling and build accurate theoretical models (based on the method of stitching) of vibration modes of a dynamic system of control of physical and mechanical properties of materials, the design of which provides periodic contact with the product. Such regimes are not connected with the need of excitation of the modes of wave oscillations directly in the product. There is a need of developing a special vibratory devices with high precision and speed control of mechanical properties when equipped with such devices special elements based on the surface of the product.

В практике физических, физико-технических измерений и испытаний осваиваются вибрационные неразрушающие методы. Одним из важнейших направлений определения качества промышленной продукции является оценка свойств материалов неразрушающими методами на основе вибрационных, акустических, ультразвуковых и других видов колебаний. Подобные методы реализуются не только в средствах контрольной и измерительной техники, но и находят применение для создания новых технологических процессов. 
Актуальным является повышение эффективности методов и средств неразрушающего контроля физико-механических свойств материалов $\mathrm{c}$ помощью преобразователей, подвижные системы которых обеспечивают периодический контакт с изделием. Такие режимы не связаны с необходимостью возбуждения мод волновых колебаний непосредственно в самом изделии. В настоящее время появилась необходимость разработки специальных вибрационно-контактных устройств, обладающих высоким быстродействием контроля механических свойств при оснащении их специальными элементами базирования на поверхности изделия. Вибрационноконтактные устройства имеют режимы в диапазоне возбуждения низких частот механических колебаний, что обеспечивает возможность их использования для контроля не только неподвижных, но и движущихся изделий. На резонансных режимах колебаний обеспечивается получение сравнительно больших рабочих амплитуд измерительного наконечника. При этом повышение магнитной проводимости генераторной системы вибрационно-контактного преобразователя позволяет существенно повысить ее чувствительность.

Для контроля твердости изделий из металлов, а также изделий из других видов материалов, имеющих широкий диапазон значений модуля упругости, может использоваться одномерный вибрационно-контактный преобразователь (рис.1), снабженный мультивибратором 1, вибрирующим щупом 2 и генераторной системой 3. С этой целью преобразователь оснащается элементами его базирования 4 на изделии 5.

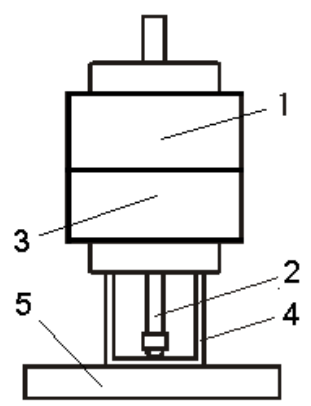

Рис.1.Схема преобразователя 
В данной работе исследуются периодические резонансные режимы, в которых измеряемый объект является элементом динамической системы. Поэтому представляют интерес задачи, связанные с созданием не только моделей адекватного аналитического описания движения подобных систем, но и моделей контроля физико-механических свойств металлических и других изделий в том числе. При этом восстанавливающие силы, действующие на измерительный наконечник, являются нелинейными и описываются соответствующими кусочно-линейными функциям, обусловленными существенным различием жесткости упругого подвеса и контактной жесткости поверхности изделия. Периодический режим измерительного наконечника относительно поверхности контролируемого изделия не является ударным, при котором длительность соударения принимается равной нулю, а эффект соударения оценивается с помощью коэффициента восстановления скорости. Процесс вибрационно-контактного контроля твердости рассматривается как процесс конечной длительности, соответствующей установившемуся режиму колебаний. Рассмотрим вибрационно-контактную систему, имеющую характеристику восстанавливающей силы, обусловленную значительным различием жесткости в зоне и вне зоны контакта.

Следовательно, соответствующую динамическую характеристику $f(x)$, реализуемую в решениях уравнений движения одномерной системы с помощью приближенного метода гармонического баланса, представим в виде

$$
f(x)=c_{1} x, x \leq \Delta ; c_{1} \Delta+\left(c_{1}+c_{2}\right)(x-\Delta), x \geq \Delta,
$$

где с1 и с2 - жесткости упругого подвеса и объекта контроля соответственно; $\Delta$ - расстояние от измерительного наконечника до поверхности объекта контроля. Динамическая характеристика $f(x)$ представлена кусочнолинейными функциями, отражающими результат линеаризации гладких или кусочно-гладких зависимостей воспроизведения физико-механических свойства нелинейной динамической системы. Линеаризованные выражения 
восстанавливающих сил должны отвечать решениям уравнений движения в соответствии с принятым методом исследования.

Анализ амплитудно-частотных зависимостей и результатов экспериментальных исследований показал, что контроль локальных физикомеханических свойств изделий целесообразно производить или в режиме вынужденных колебаний, или на частоте основного резонанса, т.е. при фиксированной под конкретную подвижную систему частоте возмущающей силы. Во втором случае упрощается не только математическая модель колебаний, но и появляется возможность определения влияния жесткости контролируемого изделия на амплитуду и смещение центра колебаний системы относительно положения статического равновесия при меньшем числе варьируемых параметров. Математическую модель для построения точного решения колебаний в режиме вибрационного контакта без демпфирования представим в безразмерных переменных

$$
\ddot{\xi}+f(\xi)=\eta \cos \tau
$$

где безразмерные переменные и варьируемые параметры представлены следующими выражениями:

$$
\xi=x \Delta^{-1} ; \eta=H\left(m \Delta \omega^{2}\right)^{-1},
$$

где $\mathrm{x}$ - абсолютная величина перемещения измерительного наконечника относительно его положения статического равновесия;

Н - амплитуда возмущающей гармонической силы;

$\omega$ - круговая частота возмущающей силы;

$\mathrm{t}$ - время.

Уравнение (2) описывают две фазы процесса периодического взаимодействия: фазу контакта и фазу отскока измерительного наконечника. Рассматриваемому режиму установившихся колебаний измерительного наконечника на фазовой плоскости соответствует замкнутая фазовая траектория. При этом время пробега изображающей точкой фазовой траектории совпадает с 
периодом возмущающей силы. Указанная фазовая траектория состоит из двух участков:

участок траектории, соответствующей движению измерительного органа в контакте с поверхностью измеряемого изделия;

участок траектории, соответствующей движению измерительного органа без контакта с поверхностью измеряемого изделия.

Предельные отклонения измерительного наконечника $\xi_{+} \xi_{-}$позволяют установить аналитическую связь между амплитудой, смещением центра колебаний и жесткостью измеряемого изделия.

Шкала отсчетного устройства вибрационно-контактного преобразователя может быть аттестована в единицах твердости. В данном случае необходимо жесткость изделия выразить через жесткость меры твердости, естественно, в единицах твердости шкал Бринелля, Роквелла, Виккерса, Шора.

C помощью предложенной математической модели установлена зависимость между механическими свойствами и амплитудой вибрационноконтактной системы, а также предсказана возможность осуществления контроля указанных свойств.

Рассмотрено исследование вибрационно-контактной системы и еe режимов вынужденных колебаний с помощью аппарата точных аналитических зависимостей.

\section{Литература:}

1. Вибрации в технике. Защита от вибраций и ударов; Т.6: Справочник / Под ред. К.В. Фролова/. М.: Машиностроение, 1981. - 456 с.

2. Федотов, А.И. Теория измерений /А.И. Федотов, С.К. Лисин, Г.С. Морокина. - СПб.: Изд-во Политех. ун-та, 2013. - 325 с.

3. Victor Wowk. Machinery Vibration: Measurement and Analysis. McGraw - Hill Professional, 1991, 358 p. 